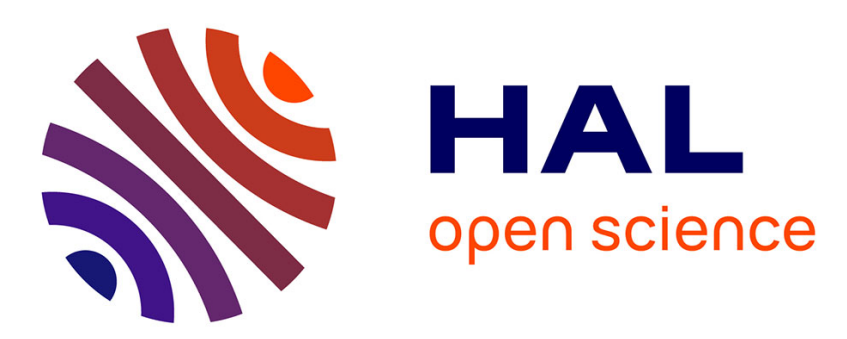

\title{
A global hybrid model for Titan's interaction with the Kronian plasma: Application to the Cassini Ta flyby
}

Ronan Modolo, Gérard M. Chanteur

\section{To cite this version:}

Ronan Modolo, Gérard M. Chanteur. A global hybrid model for Titan's interaction with the Kronian plasma: Application to the Cassini Ta flyby. Journal of Geophysical Research Space Physics, 2008, 113 (A10), pp.A01317. 10.1029/2007JA012453 . hal-00365082

\section{HAL Id: hal-00365082 https://hal.science/hal-00365082}

Submitted on 4 Feb 2016

HAL is a multi-disciplinary open access archive for the deposit and dissemination of scientific research documents, whether they are published or not. The documents may come from teaching and research institutions in France or abroad, or from public or private research centers.
L'archive ouverte pluridisciplinaire HAL, est destinée au dépôt et à la diffusion de documents scientifiques de niveau recherche, publiés ou non, émanant des établissements d'enseignement et de recherche français ou étrangers, des laboratoires publics ou privés. 


\title{
A global hybrid model for Titan's interaction with the Kronian plasma: Application to the Cassini Ta flyby
}

\author{
R. Modolo ${ }^{1,2}$ and G. M. Chanteur ${ }^{1}$ \\ Received 5 April 2007; revised 24 August 2007; accepted 28 September 2007; published 31 January 2008.
}

[1] The interaction between the corotating magnetospheric plasma of Saturn and the exosphere of Titan is investigated by means of a three-dimensional and multispecies hybrid simulation model coupling charged and neutral species via three ionizing mechanisms: the absorption of extreme ultraviolet solar photons, the impacts of magnetospheric electrons, and the charge exchange reactions between ions and neutral atoms or molecules. The simulation model includes the low and energetic components of the magnetospheric plasma, the main exospheric neutral species (molecular hydrogen and nitrogen and methane), and the atmospheric slowing down of charged particles penetrating below the exobase. Ionization rates of the exospheric species are computed as consistently as possible for each of the three ionizing mechanisms by making use of the relevant local number densities and cross sections or ionization frequencies. This model is thus able to provide a priori estimates of the escaping fluxes of exospheric ionic species and to separate for the contributions of the different ionization sources. A simulation run has been made for the conditions encountered by spacecraft Cassini during flyby Ta of Titan on 26 October 2004. Results are presented to characterize the main features of the simulated plasma environment of Titan: the induced magnetic tail and the flow of magnetospheric plasma around Titan, as well as the wake and the acceleration of the planetary plasma. Considering the coarse spatial resolution of the present simulation, these features are in reasonable agreement with in situ plasma measurements made by spacecraft Cassini.

Citation: Modolo, R., and G. M. Chanteur (2008), A global hybrid model for Titan's interaction with the Kronian plasma: Application to the Cassini Ta flyby, J. Geophys. Res., 113, A01317, doi:10.1029/2007JA012453.

\section{Introduction}

[2] The Saturnian system provides a variety of interesting interactions of the magnetospheric plasma with the rings, the icy satellites or the other moons. The Cassini-Huygens mission offers the possibility to investigate these different interactions and explore the plasma environment of Saturn [cf. Blanc et al., 2002]. This study focuses on the interaction of Titan with the surrounding magnetospheric plasma, one of the major objectives of the mission. This interaction presents specific features which make it a unique case in the solar system.

[3] The plasma interaction at Titan depends upon several parameters. The orbit of Titan, located at 20.3 Saturn radii, is most of the time within Saturn's magnetosphere. The

\footnotetext{
${ }^{1}$ Centre d'Etudes des Environnements Terrestre et Plantaires, Institut Pierre Simon Laplace, Vélizy, France.

${ }^{2}$ Swedish Institute of Space Physics, Uppsala, Sweden.

Copyright 2008 by the American Geophysical Union. 0148-0227/08/2007JA012453
}

stand-off distances of the bow shock and of the magnetopause are controlled by the dynamic pressure of the solar wind, and under peculiar circumstances they can be such that Titan may be found in the magnetoseath of Saturn or even in the solar wind [Wolf and Neubauer, 1982]. The ambient plasma in these regions differs by its ion composition and by the nature of its flow. Inside the magnetosphere the plasma flow around Titan has an Alfvènic Mach number of the order of 1 and a sonic Mach number lower than one. This interaction is thus qualitatively different from the solar wind plasma interaction at Mars, Venus, or comets, characterized by hypersonic and hyper-Alfvenic $\left(\mathrm{M}_{S} \gg 1\right.$ and $\mathrm{M}_{A} \gg 1$ ) incoming flows.

[4] Titan has a cold, dense, and thick atmosphere composed of molecular nitrogen and methane mixed together with minor species [Yung, 1987; Toublanc et al., 1995; Yelle et al., 2006]. The thickness of this atmosphere is worth noticing: with an exobase located at an altitude of $\sim 1430 \mathrm{~km}$ according to Waite et al. [2005].

[5] The atmosphere and the ionosphere of Titan are not protected by an intrinsic magnetic field [Backes et al., 2005] and the plasma interaction is thus mostly of the atmospheric type [Neubauer et al., 1984]. The location and strength of 
each ionization source depends upon the orbital phase of Titan around Saturn; thus the various ionization sources are usually not spatially coincident.

[6] Several models of Titan's interaction with the incoming plasma has been developed since Voyager-1 observations in 1980. The multispecies magnetohydrodynamic approach have been used in most of the one-dimensional [Keller et al., 1994], two-dimensional [Cravens et al., 1998], and threedimensional models [Ledvina and Cravens, 1998; Kabin et al., 2000; Nagy et al., 2001; Backes et al., 2005; Ma et al., 2006]. Among these MHD models, that one developed by Backes et al. [2005] involves a detailed description of the magnetospheric and ionospheric electrons, providing a good agreement with the magnetic field observations around the closest approach during the Ta flyby. Very good concordances for the Ta and Tb flybys are also found by Ma et al. [2006] with models involving a set of chemistry equations to describe the ionosphere. These models provide a global description of the plasma environment in Titan's close vicinity but are not able to take into account ion gyroradii effects which cannot be ignored in remote regions beyond the exobase, since the Larmor radii of the $\mathrm{O}^{+}$ambient ions, as well as the gyroradii of the heavy pickup ions, are larger than the radius of the satellite itself. The importance of finite gyroradii effects near Titan has been first investigated by means of test particle simulations [Luhmann, 1996; Ledvina et al., 2005].

[7] A proper description of these kinetic effects requires at least the use of hybrid models which combine a kinetic dynamics of the ionic species with a fluid description of the electrons. Such semikinetic models have been recently developed for the plasma interaction at Titan by Brecht et al. [2000], Kallio et al. [2004], Sillanpää et al. [2006], and by Simon et al. [2006]. All these models show the importance of finite gyroradii effects at a few Titan radii, and most of them emphasize the necessity of a multispecies approach. The hybrid model presented here differs from the preceding ones by the distinction made between the various ionization sources, a detailed consideration of the dynamics of the energetic kronian plasma as well as of the dynamics of the $\mathrm{H}_{2}^{+}$ions. This simulation model includes ionization by extreme ultraviolet solar photons, by impacts of magnetospheric electrons and by charge exchange reactions. The neutral environment is described by the three main exospheric species: methane, molecular nitrogen, and molecular hydrogen. The production rates associated to the ionization sources are calculated locally and self-consistently by making use of the neutral densities and relevant ionization frequencies or cross sections. The same approach for the calculation of the production rates has been used successfully to describe the solar wind interaction with Mars [Modolo et al., 2005, 2006]. Moreover, this simulation model takes into account for the first time six ionic species: the kronian plasma is modeled by thermal $\mathrm{O}^{+}$and $\mathrm{H}^{+}$, and energetic $\mathrm{H}^{+}$, meanwhile the planetary plasma created by the ionization of the neutral exosphere involves three additional ionic species $\left(\mathrm{CH}_{4}^{+}, \mathrm{N}_{2}^{+}\right.$, and $\left.\mathrm{H}_{2}^{+}\right)$. We focus this investigation on flyby $\mathrm{Ta}$ of Titan by spacecraft Cassini which occurred on 26 October 2004. This paper is organized as follows: section 2 presents the main features of the simulation model, section 3 presents some simulation results, especially the description of the draping of the magnetic field around Titan (section 3.1), the plasma environment (section 3.2), the acceleration of the planetary plasma (section 3.3), and estimates of the escaping ionic fluxes (section 3.5). Results from the simulation are compared to the main features of the observations made by Voyager-1 and Cassini during flyby Ta.

\section{Description of the Model}

[8] The present three-dimensional model belongs to the large class of hybrid models which retain a fully kinetic description of ions and treat the electrons as an inertialess fluid contributing to electric currents and enforcing charge neutrality. Particles and fields are treated self-consistently.

[9] Ions are represented by a set of macroparticles obeying the laws of motion of physical particles:

$$
\begin{gathered}
\frac{d \mathbf{v}_{j}}{d t}=\frac{q_{j}}{m_{j}}\left(\mathbf{E}+\mathbf{v}_{j} \times \mathbf{B}\right) \quad\left\|\mathbf{x}_{j}-\mathbf{x}_{\text {planet }}\right\|>R_{T}+h_{\text {exobase }} \\
\frac{d \mathbf{v}_{j}}{d t}=\frac{q_{j}}{m_{j}}\left(\mathbf{E}+\mathbf{v}_{j} \times \mathbf{B}\right)-\mathbf{F}_{c} \quad\left\|\mathbf{x}_{j}-\mathbf{x}_{\text {planet }}\right\| \leq R_{T}+h_{\text {exobase }} \\
\frac{d \mathbf{x}_{j}}{d t}=\mathbf{v}_{j}
\end{gathered}
$$

where $\mathbf{x}_{j}, \mathbf{v}_{j}, q_{j}$, and $m_{j}$ are the position, velocity, charge, and mass, respectively, of macroparticle $\mathrm{j}$. Here $\mathbf{x}_{\text {planet }}, R_{T}$, and $h_{\text {exobase }}$ are the position of the planet in the simulation grid, the radius of Titan, and the altitude of the exobase, while $F_{c}$ is the drag force exerted by the atmosphere on the charged particle; this way of taking atmospheric collisions into account is discussed in section 2.2. The total number of macroparticles can be very large (several millions for simulations of planetary environments) and can be time dependent. A macroparticle can be viewed as a cloud of a given number of identical physical particles travelling together at exactly the same velocity: it has no internal degree of freedom; thus it cannot be considered as an elementary volume of plasma. Owing to their finite size and their ability to interpenetrate each other, macroparticles do not suffer close binary collisions and thus are able to mimic the Debye shielding occurring in plasmas. This basic property is the main justification of this concept. The number of physical particles represented by a given macroparticle is named its statistical weight. In uniform plasmas, using identical statistical weights for chemical species having very different physical densities would lead to insufficient statistical sampling of the minor species. Similarly, the use of identical statistical weights for macroparticles belonging to a given species having large spatial gradients would lead to insufficient statistics in the low-density regions or to extremely large number of macroparticles in dense regions. The use of macroparticles having individual statistical weights allows to overcome these difficulties. The assumption of a massless and charge neutralizing fluid, leading to $n_{e}=\sum_{i} n_{i}=\rho$, is valid for scale lengths greater than the Debye length, which is the case for scale lengths considered in this study. Hence the 
electric field is a function of state computed from the electron momemtum equation:

$$
\mathbf{E}=-\frac{\mathbf{J}_{i} \times \mathbf{B}}{\rho}+\frac{(\nabla \times \mathbf{B}) \times \mathbf{B}}{\mu_{0} \rho}-\frac{\nabla P_{e}}{\rho}
$$

where the ionic current density $\left(\mathbf{J}_{i}=\sum_{i} n_{i} q_{i} \mathbf{U}_{i}\right)$ is computed from the macroparticle data (density $n_{i}$, charge $q_{i}$, and velocity $\left.\mathbf{U}_{i}\right)$ and the total current density $\left(\mathbf{J}=\mathbf{J}_{e}+\mathbf{J}_{i}\right)$ is derived from Ampère's equation in which the displacement current term is neglected owing to the fact that only lowfrequency phenomena are of interest. The time evolution of the magnetic field results from Faraday's equation, meanwhile satisfying the solenoidal condition:

$$
\begin{gathered}
\frac{\partial \mathbf{B}}{\partial t}=-\nabla \times \mathbf{E} \\
\nabla \cdot \mathbf{B}=0
\end{gathered}
$$

$\mathbf{E}$ and $\mathbf{B}$ are computed on two identical grids shifted each other by half a grid size in all directions and allow ensuring the divergence free of the magnetic field to the second-order approximation [Birdsall and Langdon, 1985]. Different schemes exist to solve the huge system of coupled equations (1) to (6) governing the motion of particles and the evolution of the electromagnetic field. The core of the present hybrid model is founded on the "Current Advance Method and Cyclic Leapfrog" algorithm designed by Matthews [1994], now frequently referred as CAMCL. This latter reference gives a detailed description of the CAMCL algorithm and of its validation tests. The CAMCL kernel has been redesigned for the modelling of planetary environments [Modolo, 2004; Modolo and Chanteur, 2005]. First, by implementing individual statistical weights of macroparticles to enable a large range of physical densities extending over more than 6 decades. Second, by designing and implementing sources and sinks of particles and open boundary conditions adapted either to subsonic or hypersonic flows of the external plasma. Third, by optimizing the use of computer memory despite the fluctuating numbers of macroparticles resulting from the creation and loss of particles, and from open boundaries.

[10] The model for the plasma interaction at Titan shares these generic features with the simulation model developed to study the solar wind interaction with the Martian exosphere [Modolo et al., 2005; Modolo and Chanteur, 2005; Modolo et al., 2006]. The first version of this model has been presented by Chanteur and Modolo [2005], it already included all the ionization sources known to be effective in Titan's environment (especially the contribution from the corona of molecular hydrogen), but when the observations of Cassini during flyby Ta were announced that lead to some difficulties ignored by less exhaustive models existing at that time [e.g., Kallio et al., 2004]. It happened that with the plasma parameters derived from Cassini observations, the magnetospheric plasma was not able to confine the planetary plasma. That lead to a weak but noticeable nonstationarity due to a slight but steady inflation of the domain filled by the planetary plasma [Chanteur and Modolo, 2005]. The present simulation model for Titan's environment is enriched with respect to the earlier one by several important processes such as the atmospheric breaking of charged particles penetrating below the exobase (section 2.2), the photoabsorption when computing the photoproduction (section 2.3), the description of the energetic magnetospheric plasma (section 2.4), and the calculation of the momentum transfer during charge exchange collisions between heavy incident ions $\left(\mathrm{O}^{+}\right)$and light neutral molecules $\left(\mathrm{H}_{2}\right)$.

[11] The coordinate system used is centered on Titan: the $\mathrm{X}$ axis is parallel to the velocity $\mathbf{V}$ of the corotating plasma flow; the $\mathrm{Y}$ axis is orthogonal to $\mathbf{V}$, lays in the orbital plane of Titan and points toward Saturn; the $\mathrm{Z}$ axis completes the right-handed system and thus is parallel to the spin axis of Saturn. The size of the simulated domain is $-6.4 \leq X \leq 7.4$ Titan radii $\left(R_{T}\right)$ and $-10.8 \leq Y, Z \leq 10.8 R_{T}$. The simulation domain is meshed by a three-dimensional (3-D) uniform cartesian grid with a spatial resolution of $500 \mathrm{~km}$. The dense atmosphere of Titan is considered as a fully absorbing obstacle; particles reaching or penetrating below an altitude of $700 \mathrm{~km}$ are stopped (but not removed from the simulation) and thus cease to contribute to the evolution of the system. Let us remark that below the exobase, located at $1430 \mathrm{~km}$ above the surface [Waite et al., 2005], the model cannot provide a rigorous description of the plasma since the complex ionospheric chemistry is not included. Efficient models have been developed to describe Titan's ionosphere [Toublanc et al., 1995; Keller et al., 1998; Galand et al., 1999; Cravens et al., 2005, and references within]. However, the slowing down of the ions below the exobase can be roughly described by using the stopping power of the various atmospheric components (see section 2.2). Despite its simplicity the fluid model used to describe the electrons discriminates the hot electrons of the external plasma from the colder electrons of the planetary plasma by making at each timestep a partition of the simulation domain according to the instantaneous location of the transition boundary between these two plasmas: an adiabatic behavior determined by a polytropic index equal to 2 is assumed in each subdomain, with two different references of temperature for the undisturbed external plasma and for the ionospheric plasma. This physical and dynamic partition has been first designed and implemented in our Martian model [Modolo et al., 2005; Modolo and Chanteur, 2005; Modolo et al., 2006] and proved to be an essential feature of the modelling. A similar approach has been adopted but implemented differently by Simon et al. [2006].

[12] No particular condition on the electromagnetic field is imposed inside the obstacle. Periodic boundary conditions along $\mathrm{Y}$ and $\mathrm{Z}$ directions, perpendicular to the direction of the external flow, are applied to the electromagnetic field and to macroparticles describing the external plasma. Open boundary conditions are used in the $\mathrm{X}$ direction at the entry and exit faces of the magnetospheric plasma. Modolo et al. [2005] have described boundary conditions which can be implemented in a kinetic simulation model for a supersonic flow of the external plasma, but simulating a subsonic flow is a more difficult task. In this latter case, particles need to be injected not only through the entry face of the simulation box (at $X=-6.4 R_{T}$ ) but also through the exit face (at $X=7.4 R_{T}$ ) with a "physically reasonable" velocity distribution function. The difficult point is that the velocity distribution function of a plasma flow is unknown down- 
Table 1. Parameters of the Piecewise Isothermal Profiles for the Three Exospheric Species Taken Into Account by the Simulation Model $^{\mathrm{a}}$

\begin{tabular}{lccc}
\hline Species & Altitude, $\mathrm{km}$ & Density, cm & Temperature, $\mathrm{K}$ \\
\hline $\mathrm{N}_{2}$ & 700. & $7.2 \times 10^{11}$ & 159 \\
& 1265. & $2.6 \times 10^{8}$ & 149 \\
$\mathrm{CH}_{4}$ & 925. & $7.2 \times 10^{8}$ & 109 \\
& 1265. & $7 . \times 10^{6}$ & 149 \\
$\mathrm{H}_{2}$ & 700. & $2 . \times 10^{9}$ & 110 \\
& 1400. & $6 . \times 10^{5}$ & 149 \\
\hline
\end{tabular}

${ }^{a}$ Each isothermal layer is defined by its temperature and its base level Number densities are imposed at the lower base level, and they are indicated together with the computed number densities at the second base level.The density of methane below $925 \mathrm{~km}$ is fixed to $2 \%$ of the density of molecular nitrogen.

stream of such a complex obstacle as a planetary body. There is unfortunately no alternative to using the velocity distribution function of the undisturbed external plasma flow, meanwhile placing the injection boundaries as far away as possible from the perturbing obstacle. In order to damp inward penetrating perturbations generated near the exit face, a spatial smoothing of the electromagnetic field is applied on the last five planes before the exit face. Indeed, even without considering the specific problem of particle injection, this golden rule of placing the boundaries as far away as possible from the interaction region is applied in all directions in order to minimize pressure perturbations reaching the boundaries. Open boundary conditions are applied to all components of the planetary plasma which is allowed to leave freely the simulation domain through any of its edges.

\subsection{Titan's Exosphere}

[13] In our model the neutral environment of Titan is modeled by three spherically symmetric coronae of methane, molecular hydrogen and molecular nitrogen, the three major components of Titan's exosphere [Waite et al., 2005; Yelle et $a l ., 2006]$. An atomic hydrogen corona exists but is less dense than the molecular hydrogen corona by almost a factor 10 [Toublanc et al., 1995; Garnier et al., 2007]. In addition $\mathrm{H}^{+}$ production rates (by solar photons, by electronic impact or charge exchange reactions) are smaller than the $\mathrm{H}_{2}^{+}$production rates (except for the production by charge exchange reaction between thermal protons and atomic hydrogen). In a first attempt the $\mathrm{H}^{+}$production rates, and so the atomic hydrogen corona, have not been included in the simulation model. Several models have been developed to describe Titan's atmosphere [Yung et al., 1984; Toublanc et al., 1995; Lebonnois et al., 2001] but they are mainly focused on the lower atmosphere and do not describe the upper atmosphere above $2000 \mathrm{~km}$, where the creation of pickup ions takes place. We chose radial profiles of densities given by simplified Chamberlain's models [Chamberlain, 1963]: each neutral species is represented by a fluid in pressure equilibrium in the gravity field of the satellite but in two superposed isothermal layers having two different temperatures instead of a unique isothermal layer covering the full range of altitudes. The partial pressure $p_{i}$ of chemical species " $i$ " is solution of the following differential equation:

$$
\frac{d p_{i}}{d r}=-\frac{\mathcal{G} M_{T}}{r^{2}} \frac{M_{i} p_{i}}{R T_{i}}
$$

where $\mathcal{G}$ is the gravitational constant, $M_{T}$ is the mass of Titan, $M_{i}$ and $T_{i}$ are the molar mass and temperature of the neutral species " $\mathrm{i}$," and $\mathrm{R}$ is the constant of gases. For each species the differential equation is integrated for given piecewise-constant temperature profiles with given number densities at the lower altitude. Table 1 presents the temperatures and the densities used to compute the densities profiles of the three neutral species taken into account. Below $925 \mathrm{~km}$, the density of methane has been fixed to $2 \%$ of the molecular nitrogen density, in agreement with the Ion and Neutral Mass Spectrometer (INMS) observations [Waite et al., 2005; Yelle et al., 2006].

[14] Figure 1 shows the radial density profiles of the neutral exospheric species of Titan taken into account by the model for altitudes greater than $700 \mathrm{~km}$. Methane and molecular nitrogen are dominant below $2000 \mathrm{~km}$. Molecular hydrogen, owing to its low mass, is the dominant specie above $2000 \mathrm{~km}$. The description of the corona of molecular hydrogen is important to characterize the interaction the kronian plasma with the neutral environment of Titan, this is a substantial source of planetary ions and a corresponding loss of atmospheric neutrals. The density profiles of molecular nitrogen and methane are in good agreement, at least up to $1500 \mathrm{~km}$, with recently published models, derived from the Voyager-1 observations [Vervack et al., 2004], and from the latest results of INMS [Waite et al., 2005]. The density profile of $\mathrm{H}_{2}$ is similar to the profile derived by Amsif et al. [1997] and is in agreement with the INMS observations [Yelle et al., 2006]. Temperatures and densities in the range of $1200-2000 \mathrm{~km}$ control the extension of the neutral coronae, and thus influence the ionic production rate; a better description of the upper atmosphere taking into account the day/night asymmetry should improve the estimation of the atmospheric loss.

\subsection{Atmospheric Breaking of Charged Particles}

[15] The dynamics of the ions below the exobase cannot be rigorously described with an hybrid model, a Monte Carlo simulation model would be more appropriate. Nevertheless the slowing down of the ions in the upper atmosphere can be roughly described by including the

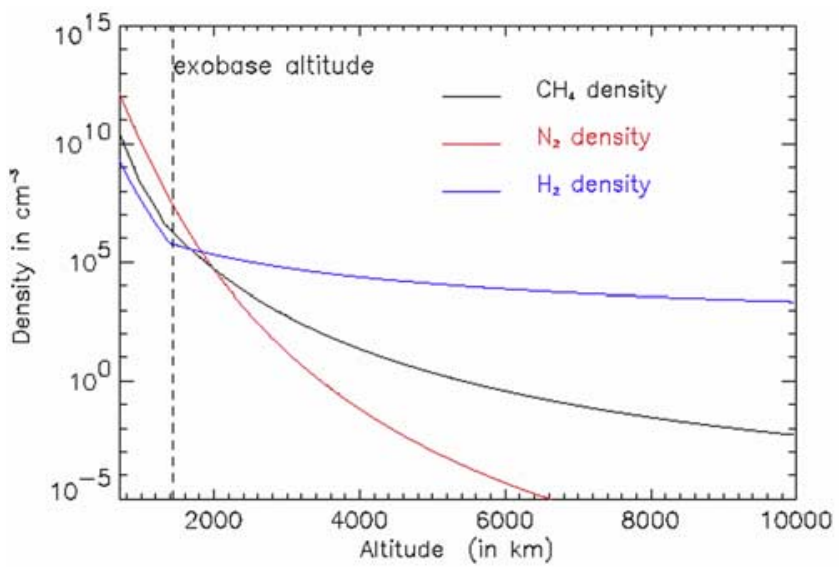

Figure 1. Radial density profiles of the exospheric neutral species used in the simulation model. Molecular nitrogen, methane, and molecular hydrogen density profiles are represented by the red, black, and blue curves, respectively. 
stopping power of the atmosphere into the model. The stopping power of matter is the average rate of energy loss per unit path length of a charged particle moving through. This loss of energy is due to Coulomb collisions resulting in ionization and excitation of atoms and molecules (electronic stopping power), to the transfer of energy to recoiling nuclei in elastic collisions (nuclear stopping power), and for light particles like electrons to radiative losses [Berger et al., 2005, and reference within]. For ions the electronic stopping power dominates the nuclear one except at very low energies below a few $\mathrm{keV}$. This concept is implemented in the simulation model by computing the energy loss of each concerned macroparticle during the time step:

$$
E_{j}(t+1)=E_{j}(t)-\Delta E
$$

where $E_{j}(t)$ is the energy of the macroparticle $j$ at time $t$ and $d E$ is the energy lost along the path of the particle during the time step.

$$
\Delta E=\sum_{s} \Delta E_{s}, \quad s=\mathrm{CH}_{4}, \mathrm{~N}_{2}
$$

$\Delta E_{s}$ is the energy loss when the macroparticle travels in a given material $s$ (here the summation is over the two main atmospheric components $\mathrm{CH}_{4}$ and $\mathrm{N}_{2}$ ). And $\Delta E_{s}$ is defined by:

$$
\Delta E_{s}=\frac{1}{\rho_{s}}\left|\frac{d E}{d x}\right|_{s}\left\langle\rho_{s}\right\rangle \Delta d
$$

where $1 / \rho_{s}|d E / d x|_{s}$ is the stopping power for material $s,\left\langle\rho_{s}\right\rangle$ is the average mass density of material $s$ along the path length $\Delta d$ travelled by the particle during the time step. For $1 \mathrm{keV}$ protons the stopping power of $\mathrm{N}_{2}$ is equal to $1.49 \times$ $10^{2} \mathrm{MeV} \mathrm{cm}^{2} \mathrm{~g}^{-1}$ and that of $\mathrm{CH}_{4}$ to $3.48 \times 10^{2} \mathrm{MeV} \mathrm{cm}^{2}$ $\mathrm{g}^{-1}$ [Berger et al., 2005]. In this first attempt to compute the atmospheric slowing down of ions, we have deliberately chosen a rather coarse and simplistic implementation involving a stopping power independent of energy and identical for all the ionic species. This was made to palliate artefacts of our earlier simulations of the plasma interaction at Titan (unpublished) in which too many planetary ions where created in the dense atmosphere well below the exobase. The time rate of energy loss experienced by an energetic ion moving at velocity $\vec{v}$ through the atmosphere $d E / d t=-|d E / d x|\|\vec{v}\|$ is equal to $\vec{F}_{c} \cdot \vec{v}$, where $\vec{F}_{c}$ is the drag force exerted by the atmosphere on the charged particle. The drag force, opposite to the velocity of the particle, is a simple way to represent the effect of numerous collisions along the path of the charged particle when neglecting the small and random angular deviations resulting from individual collisions.

[16] Results presented in section 3 demonstrate that it was worth including the effect of the dense atmosphere on the motion of charged particles; hence in future work the simulation model will be improved by distinguishing the stopping power of the different ions meanwhile retaining its dependency upon their energy.

\subsection{Ionization Processes}

[17] Planetary ions are produced by three ionization processes: photoionization, electronic impacts, and charge exchange reactions between planetary neutrals and ions, either primary ions pertaining to the magnetospheric plasma or secondary ions of planetary origin. The production rates are computed following the method designed by Modolo and Chanteur [2005] and used by Modolo et al. [2005]: they are not imposed but computed locally and self-consistently with regards to relevant neutral densities and ionization frequencies or cross sections, separately for each planetary species and each ionization process.

[18] Local photoproduction rates of $\mathrm{N}_{2}^{+}, \mathrm{CH}_{4}^{+}$and $\mathrm{H}_{2}^{+}$are calculated by making use of the Extreme Ultraviolet flux model for Aeronomics Calculations (EUVAC) developed by Richards et al. [1994] and taking into account the atmospheric photoabsorption. EUVAC is based on a reference spectrum involving 37 wavelength intervals, covering a range of 5 to $105 \mathrm{~nm}$. Table 2 gives the photoionization frequencies of the main neutral components at $1 \mathrm{AU}$ for the EUV solar flux at solar minimum and above the atmosphere, that is, not altered by the atmospheric photoabsorption. The photoabsorption by the atmosphere of the planet is computed for the chosen radial profiles of the neutral species $\mathrm{N}_{2}, \mathrm{CH}_{4}$, and $\mathrm{H}_{2}$ and by using the photoabsorption cross sections given by Schunk and Nagy [2000]. Production rates are thus calculated with the usual expression [see Schunk and Nagy, 2000]

$$
q_{X^{+}}^{\text {photo }}(z, \chi)=n_{X}(z) \int_{0}^{\lambda_{t}} \sigma_{X}^{i}(\lambda) I_{\infty}(\lambda) \exp [-\tau(z, \lambda, \chi)] d \lambda
$$

where $q_{X^{+}}$is the production rate of the ion specie $X^{+}, n_{X}(z)$ is the density of the neutral component $X$ at the altitude $z, \sigma_{X}^{i}$ is the ionization cross section of the specie $X, I_{\infty}$ is the nonattenuated solar EUV flux calculated with the EUVAC model [Richards et al., 1994] and the optical depth defined as

$$
\tau(z, \lambda, \chi) \equiv \int_{\infty}^{z} \sum_{s} n_{s}(z) \sigma_{s}^{a}(\lambda) \sec \chi d z
$$

where $\sigma^{a}$ are the absorption cross section, $\chi$ is the solar zenith angle, and index $s$ corresponds to the three main neutral species of interest $\left(\mathrm{N}_{2}, \mathrm{H}_{2}\right.$, and $\left.\mathrm{CH}_{4}\right)$. Note that the expression of the optical depth is valid for $\chi<75^{\circ}$, above this limit the full expression of the optical depth defined by Rees [1989] is used.

[19] The photoproduction rates are computed for each neutral component in the configuration encountered by spacecraft CASSINI during its first close encounter with Titan on 26 October 2004. At this date Titan was located at 1036 saturnian local time (SLT), and the declination of the Sun relative to the orbital plane of Titan was $\alpha_{\text {sol }}=23.38^{\circ}$.

[20] The electron impact ionization being an important source of planetary ions is included in this model. Only magnetospheric electron impacts are taken into account since impacts of photoelectrons do not contribute significantly to the ionization above the exobase level. Table 2 indicates the ionization frequencies per electron for a maxwellian electron gas at a temperature of $200 \mathrm{eV}$. In 
Table 2. Photoionization Frequency at $1 \mathrm{AU}$ in Solar Minimum and Electron Impacts Ionization Frequency for a Plasma With an Electronic Temperature of $200 \mathrm{eV}$

\begin{tabular}{lcl}
\hline & \multicolumn{2}{c}{ Frequency, s $^{-1}$} \\
\cline { 2 - 3 } & Photoionization & Electron Impacts \\
\hline $\mathrm{N}_{2}$ & $3.35 \times 10^{-7}$ & $16.6 \times 10^{-8}$ \\
$\mathrm{CH}_{4}$ & $5.75 \times 10^{-7}$ & $19.8 \times 10^{-8}$ \\
$\mathrm{H}_{2}$ & $7.46 \times 10^{-8}$ & $5.4 \times 10^{-8}$ \\
\hline
\end{tabular}

the present implementation of the simulation model the production rate is computed assuming that the ionization frequency per electron is independent of the electron temperature. This approximation is justified by the fact that ionization frequencies do not vary significantly for electron temperatures in the range of $150 \mathrm{eV}$ to $500 \mathrm{eV}$ (less than $10 \%$ variations). Electronic impact production rates are calculated with the following expression [Cravens et al., 1987]

$$
\begin{gathered}
q_{X^{+}}^{i m p}(\vec{r})=n_{e}(\vec{r}) n_{X}(\vec{r}) \nu_{X}^{i m p} \\
\nu_{X}^{i m p}=\int_{v_{\min }}^{\infty} v f(v) \sigma^{i m p}(v) 4 \pi v^{2} d v
\end{gathered}
$$

where $\mathrm{n}_{e}$ is the magnetospheric electron density, $\mathrm{n}_{X}$ is the neutral density of the specie $X, \mathrm{f}(\mathrm{v})$ is the normalized Maxwellian distribution function, and $\sigma^{i m p}$ is ionization cross sections by electronic impact. Cross sections used are taken from the National Institute of Standards of Technology [Kim et al., 2004].

[21] Charge exchange reactions are also included in the present simulation model. During a charge exchange collision a magnetospheric ion captures an electron from a planetary neutral atom or molecule leaving place to an energetic neutral atom (ENA) and a cold planetary ion. Production rate of planetary ions through these reactions depends upon the relevant neutral densities and cross sections and upon the relative velocity of the two colliding particles. The following reactions are considered:

$$
\left\{\begin{array}{l}
\mathrm{O}^{+} \\
H_{t h}^{+} \\
H_{\text {ener }}^{+}
\end{array}+\left\{\begin{array} { l } 
{ N _ { 2 } } \\
{ \mathrm { CH } _ { 4 } } \\
{ \mathrm { H } _ { 2 } }
\end{array} \longrightarrow \left\{\begin{array}{l}
O^{E N A} \\
H_{\text {th }}^{E N A} \\
H_{\text {ener }}^{E N A}
\end{array}+\left\{\begin{array}{l}
N_{2}^{+} \\
\mathrm{CH}_{4}^{+} \\
H_{2}^{+}
\end{array}\right.\right.\right.\right.
$$

where subscripts th and ener refer to thermal and energetic ions, respectively. So far only nondissociative charge transfer are considered, assuming that molecular bonds are not broken during the collision. Cross sections used in the simulation are given by Table 3. Variations of the considered nondissociative charge transfer cross section with the energy are poorly known in the energy range of interest $[10 \mathrm{eV}$ to $30 \mathrm{keV}]$. In a first attempt to mimic the charge exchange reactions, cross section are assumed to be constant.

[22] The required charge exchange cross sections have been extracted from different sources [Eliot, 1977; Lindsay et al., 1998; Hoffman et al., 1982; Phaneuf et al., 1978]. Only the cross sections for the $\left(\mathrm{O}^{+}, \mathrm{CH}_{4}\right)$ reaction have not been found and cross sections of the $\left(\mathrm{N}^{+}, \mathrm{CH}_{4}\right)$ reaction have been used instead.

\subsection{Magnetospheric Plasma}

[23] During the first close flyby of Titan by the Cassini spacecraft on 26 October 2004 (called $T_{A}$ flyby), observations made by experiments CAPS and MAG revealed a great variability of the magnetospheric plasma surrounding Titan, making difficult the definition of a set of parameters describing the unperturbed upstream flow of the magnetospheric plasma for the simulations. Since the observations performed by the CAPS experiment confirm the basic findings of the Voyager 1 encounter [Szego et al., 2005], we have chosen upstream parameters from the Voyager 1 encounter with Titan. Before Cassini observations the heavy ions were thought to be nitrogen ions, but Cassini/CAPS has demonstrated that heavy ions are indeed oxygen ions. In the vicinity of Titan, the background magnetopsheric plasma is mainly composed of light $\left(\mathrm{H}^{+}\right)$and heavy $\left(\mathrm{O}^{+}\right)$ions with average number densities of $0.1 \mathrm{~cm}^{-3}$ and $0.2 \mathrm{~cm}^{-3}$, respectively [Hartle et al., 1982; Neubauer, 1992]. The temperatures of the different plasma components have been chosen in accordance to the parameters observed from Voyager 1, that means $210 \mathrm{eV}$ for the thermal magnetospheric protons and $2.9 \mathrm{keV}$ for the $\mathrm{O}^{+}$magnetospheric ions [Neubauer, 1992]. The magnetospheric electron temperature has been set to $200 \mathrm{eV}$ and the ionospheric electron temperature to $1 \mathrm{eV}$. The present simulation model also differs from other models by including the energetic component of the magnetospheric plasma. This energetic component, mainly composed of energetic protons and electrons, has been characterized by the low-energy charged particle (LECP) on board Voyager 1 and 2 [Krimigis et al., 1983]. The Magnetospheric Imaging Instrument (MIMI) on board Cassini provides new insights on its spatial distribution and dynamics [Krimigis et al., 2004]. In the vicinity of Titan's orbit, Voyager 1 and 2 observations indicated a density of $1.6-1.7 \times 10^{-3} \mathrm{~cm}^{-3}$ in the energy between $16 \mathrm{keV}$ to $30 \mathrm{keV}$ [Krimigis et al., 1983]. The distribution function is well fitted by a $\kappa$ distribution with a $\kappa$ almost equal to 7 . In the present simulation of flyby $T_{A}$ these energetic protons are represented by a maxwellian population having a number density of $1.5 \times 10^{-3} \mathrm{~cm}^{-3}$ and a temperature of $30 \mathrm{keV}$. Although being a minor component with respect to the number density, the energetic electrons having energy larger than $10 \mathrm{keV}$ contributes to more than $50 \%$ of the total pressure [Maurice et al., 1996], a contribution mandatory to explain the confinement of the planetary plasma under the accepted upstream conditions for the thermal magnetospheric plasma. It is to be noticed that in our earlier, unpublished, simulations not including this

Table 3. Cross-Section Values Used for the Simulation

\begin{tabular}{llcc}
\hline & \multicolumn{3}{c}{ Cross Section, $\mathrm{cm}^{2}$} \\
\cline { 2 - 4 } & \multicolumn{1}{c}{$\mathrm{O}^{+}$} & $\mathrm{H}_{\text {th }}^{+}$ & $\mathrm{H}_{\text {ener }}^{+}$ \\
\hline $\mathrm{N}_{2}$ & $3 . \times 10^{-16}$ & $2 . \times 10^{-16}$ & $3 . \times 10^{-16}$ \\
$\mathrm{CH}_{4}$ & $5 . \times 10^{-15 a}$ & $3 . \times 10^{-15}$ & $9 . \times 10^{-16}$ \\
$\mathrm{H}_{2}$ & $1 . \times 10^{-15}$ & $2.5 \times 10^{-16}$ & $6 . \times 10^{-16}$ \\
\hline
\end{tabular}

${ }^{\mathrm{a}}$ The $\left(\mathrm{O}^{+}, \mathrm{CH}_{4}\right)$ cross section has not be found and the $\left(\mathrm{N}^{+}, \mathrm{CH}_{4}\right)$ cross section has been used. 


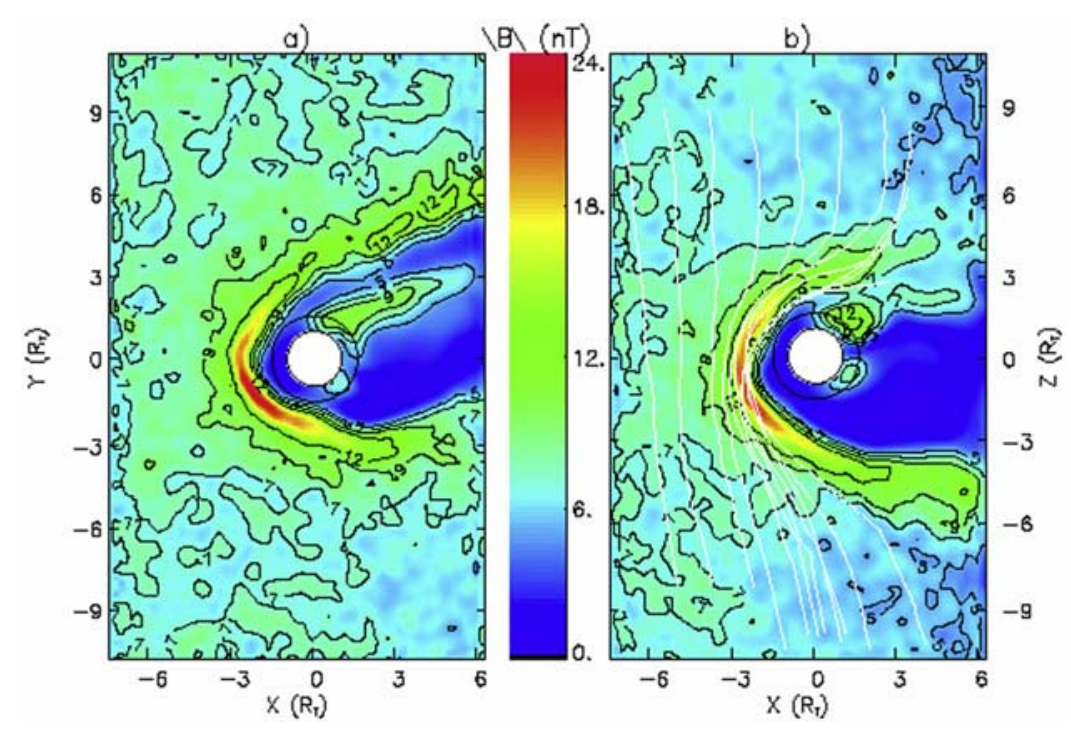

Figure 2. Maps of the total magnetic field for flyby $T_{A}$, showing (a and $\mathrm{b}$ ) the magnitude of the magnetic field in the XY and XZ planes, respectively. The black circle drawn around Titan represents the exobase located at an altitude of $1430 \mathrm{~km}$ [Waite et al., 2005] and the white circle corresponds to the surface of Titan. The white continuous lines shown in Figure $2 \mathrm{~b}$ represent projection of the magnetic field line.

energetic component, the region filled by the planetary plasma was continuously inflating, carving a correspondingly expanding cavity in the surrounding magnetospheric plasma. Under conditions of the $T_{A}$ flyby a stationary solution is reached only when this energetic plasma component is included. Since the upstream undisturbed magnetospheric plasma is not the purpose of this study, it is represented by as few macroparticles as possible (two macroparticles per cell in average for each ion species $\left(\mathrm{H}_{\text {th }}^{+}, \mathrm{H}_{\text {ener }}^{+}\right.$and $\left.\left.\mathrm{O}^{+}\right)\right)$. In order to avoid statistical problems, the same numbers of macroparticles per cell have been used for the three magnetospheric ion populations, and the statistical weights of macroparticles have been adjusted to render the physical composition of the magnetospheric plasma. The flow velocities have been chosen to be aligned with the nominal direction of the corotation and the flow speed has been set to $110 \mathrm{~km} \mathrm{~s}^{-1}$, in agreement with the Voyager 1 and Cassini observations which indicate a drift velocity in the range of $80-160 \mathrm{~km} \mathrm{~s}^{-1}$ [Hartle et al., 1982; Szego et al., 2005]. The upstream magnetic field was set as $\mathbf{B}=(0.72,2.38,-5.60) \mathrm{nT}$ in the simulation frame precedently defined, that corresponds to the average observation of the inbound magnetic field during the Ta flyby [Backes et al., 2005]. With these parameters the Alfvén velocity is equal to $74 \mathrm{~km} \mathrm{~s}^{-1}$ and the plasma $\beta$ to 8.2 . The magnetospheric flow is then super-Alfvénic and subsonic with Alfvénic and sonic mach numbers equal to 1.47 and 0.52 , respectively. These simulation parameters reflect the mean plasma parameters and the configuration encountered by Cassini during flyby $T_{A}$.

\section{Simulation Results}

[24] The simulation program is run from time $\mathrm{t}=0 \mathrm{~s}$ to $\mathrm{t} \simeq$ $2600 \mathrm{~s}$ (corresponding to 9500 time steps) and a quasistationary solution is reached around time $\mathrm{t} \simeq 1500 \mathrm{~s}$, which means that the global picture of the ionized environment is no more evolving. The simulation results are presented at time $\mathrm{t} \simeq 1500 \mathrm{~s}$ and are similar to snapshots of the state of the simulation. The time step is equal to $1 / 100 \Omega_{O+}^{-1} \simeq 0.27 \mathrm{~s}$, where $\Omega_{O^{+}}^{-1}=m_{O^{+}} / q B_{0}$ is the $\mathrm{O}^{+}$cyclotron frequency, in order to resolve accurately the cyclotron motion of the lighter protons.

[25] To discuss the main features of the three-dimensional distribution of plasma around Titan, two-dimensional cuts of the simulation output are examined: namely by plane XY containing the direction of the ideal corotation velocity $\mathbf{V}_{c o}$ $(+\mathrm{X}$ axis) and the direction toward Saturn $(+\mathrm{Y}$ axis), by plane XZ, containing $\mathbf{V}_{c o}$ and the direction of the northward spin axis of Saturn ( $+Z$ axis), and by plane $\mathrm{YZ}$, containing the direction of Saturn and the northward spin axis of Saturn. The XY plane may be referred as the equatorial plane, the XZ plane as the meridian plane (of Titan) containing the corotation velocity, the $\mathrm{XZ}$ plane is the kronian meridian plane defined by the center of Titan, it may be referred as the meridian plane (of Titan) normal to the corotation.

\subsection{Draping of the Magnetospheric Magnetic Field Around Titan}

[26] Figure 2 shows the intensity of the magnetic field in the vicinity of Titan. These maps in planes XY and XZ clearly show, according to the subsonic nature of the incoming magnetospheric flow, that a bow shock is not produced in the simulation in agreement with Cassini observations [Backes et al., 2005] and with the Voyager 1 observations [Ness et al., 1982]. The magnetic field is highly draped around Titan producing an induced magnetic tail and a pileup region on the ram side. Owing to the orientation of the incoming magnetic field, the magnetic lobes are not clearly visible on the two simulations cuts. The most intense magnetic field in the pileup region is shifted 


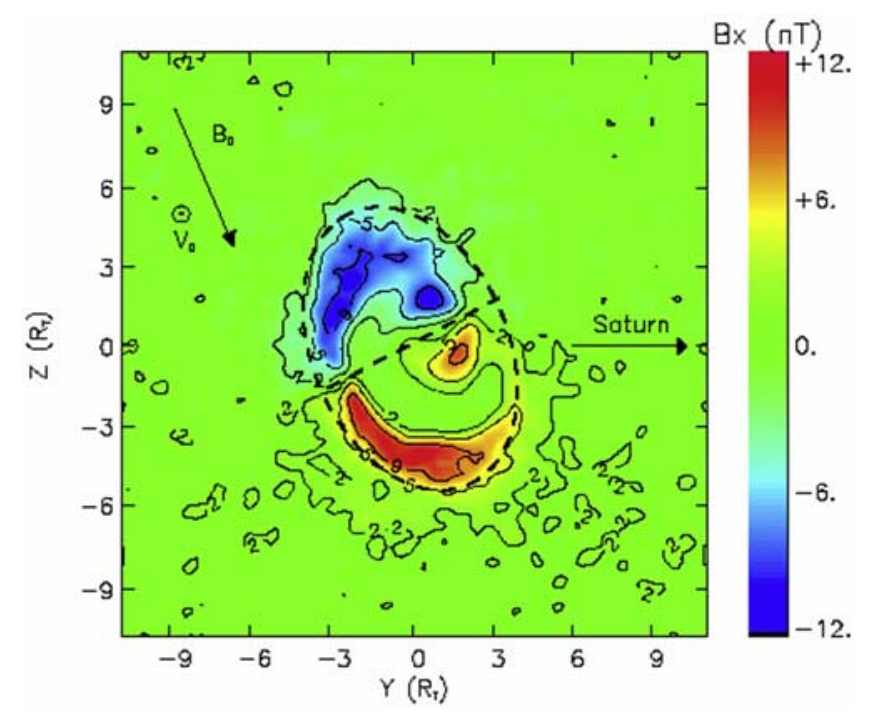

Figure 3. Map of the Bx component of the magnetic field, in the $\mathrm{YZ}$ plane located at 3 Titan radii in the wake. The $+Y$ direction points toward Saturn, and $+Z$ is parallel to the spin axis of Saturn. The projection of the upstream magnetic field is indicated as well as the direction of the incoming plasma flow.

with respect to the nominal ram direction to the side opposite to Saturn, as already noticed in other hybrid simulations [Sillanpää et al., 2006]. Moreover, a stronger magnetic field is seen on the edge of the induced magnetic tail closer to Saturn (Figure 2a), in agreement with Cassini observations [Backes et al., 2005]. These patterns have also been noticed in hybrid simulations of Sillanpää et al. [2006] but for different orbital phases. Another interesting feature is the shift of the magnetic tail toward Saturn evidenced by Figure 2a. The plasma wake of Titan is shifted in the direction opposite to the corotational electric field $(\mathbf{E}=$ $-\mathbf{V} \times \mathbf{B})$ and then, mainly directed toward Saturn. This shift of the magnetic and plasma tail have been seen first by the Voyager 1 observations [Ness et al., 1982; Hartle et al., 1982] and is very likely associated to a kinetic behavior. Sillanpää et al. [2006] suggest that as heavy ions are accelerated in the direction of the electric field, the thermal plasma flow has to move in the opposite direction, that is, toward Saturn in this case, in order to conserve the momentum transverse to the direction of the corotation. Owing to the kinetic nature of this effect, the plasma shift can not be reproduced by MHD simulations models but is selfconsistently included in hybrid models [Kallio et al., 2004; Sillanpää et al., 2006; Simon et al., 2006].

[27] Figure 3 presents the Bx component in the $\mathrm{YZ}$ plane, at three Titan radii in the wake of Titan. Figure 3 shows the cross section of the magnetic tail delimited by the ellipse overlaid on the map and reveals two magnetic lobes created by the draping of the magnetic field around the obstacle. The two lobes have an opposite polarity and are separated by a thin neutral sheet. The tilt of the magnetic wake with respect to the $Z$ axis is controlled by the direction of the upstream magnetospheric field observed by the MAG experiment on board Cassini [Backes et al., 2005]. This wake structure is mainly populated by heavy ions originating from the ionization of the neutral atmosphere of Titan (see section 3.2.2).

\subsection{Plasma Environment}

[28] The magnetospheric plasma in corotation with Saturn and the planetary plasma created by the partial ionization of the neutral exosphere constitute the plasma environment of Titan. The corotating (or kronian) plasma is mainly composed of low-energy $\mathrm{O}^{+}$and $\mathrm{H}^{+}$ions and of less than $1 \%$ of energetic protons, whereas the exospheric plasma is made of $\mathrm{N}_{2}^{+}, \mathrm{CH}_{4}^{+}$, and $\mathrm{H}_{2}^{+}$ions. Figure 4 shows density maps for $\mathrm{O}^{+}$, low-energy (or thermal) $\mathrm{H}^{+}, \mathrm{CH}_{4}^{+}$, and $\mathrm{H}_{2}^{+}$, in two-dimensional cuts of the simulation domain by $\mathrm{XY}$ and XZ planes. Density maps in Figures $4 \mathrm{c}, 4 \mathrm{e}$, and $4 \mathrm{~g}$ reveal a lateral shift of the low-energy plasma wake toward Saturn (i.e., along the $\mathrm{Y}$ axis) already noticed for the induced magnetic tail. By contrast, the plasma wake is almost symmetric in the XZ plane (Figures $4 \mathrm{~b}, 4 \mathrm{~d}, 4 \mathrm{f}$, and $4 \mathrm{~h}$ ).

\subsubsection{Kronian Plasma}

[29] Figures $4 \mathrm{a}, 4 \mathrm{~b}, 4 \mathrm{c}$, and $4 \mathrm{~d}$ show density maps of $\mathrm{O}^{+}$ and thermal $\mathrm{H}^{+}$ions in the $\mathrm{XY}$ and $\mathrm{XZ}$ planes. Upstream of Titan the density of these two species fluctuate around the $0.2 \mathrm{~cm}^{-3}$ and $0.1 \mathrm{~cm}^{-3}$, respectively. The rather large amplitude of these fluctuations is due to the small number of macroparticles per cell used to represent the unperturbed flow of these two populations. It appears from these maps that the interaction with Titan deflects the components of the magnetospheric plasma flow in two different ways. A cavity colocated with the induced magnetic tail is carved by the planetary plasma in the flow of the low-energy protons as evidenced by Figures $4 \mathrm{c}$ and $4 \mathrm{~d}$. Thermal protons are repelled from Titan by the planetary plasma at least beyond $2 \mathrm{R}_{T}$. The number density of magnetospheric thermal protons inside this cavity is lower than $1 . \times 10^{-2}$ ions $\mathrm{cm}^{-3}$. By way of contrast, Figure 4a indicates that the flow of oxygen ions is not deflected toward Saturn along the magnetic tail. Density maps of energetic protons being very much alike are not shown here, but it should be noticed that the density of this energetic population is smaller by a 

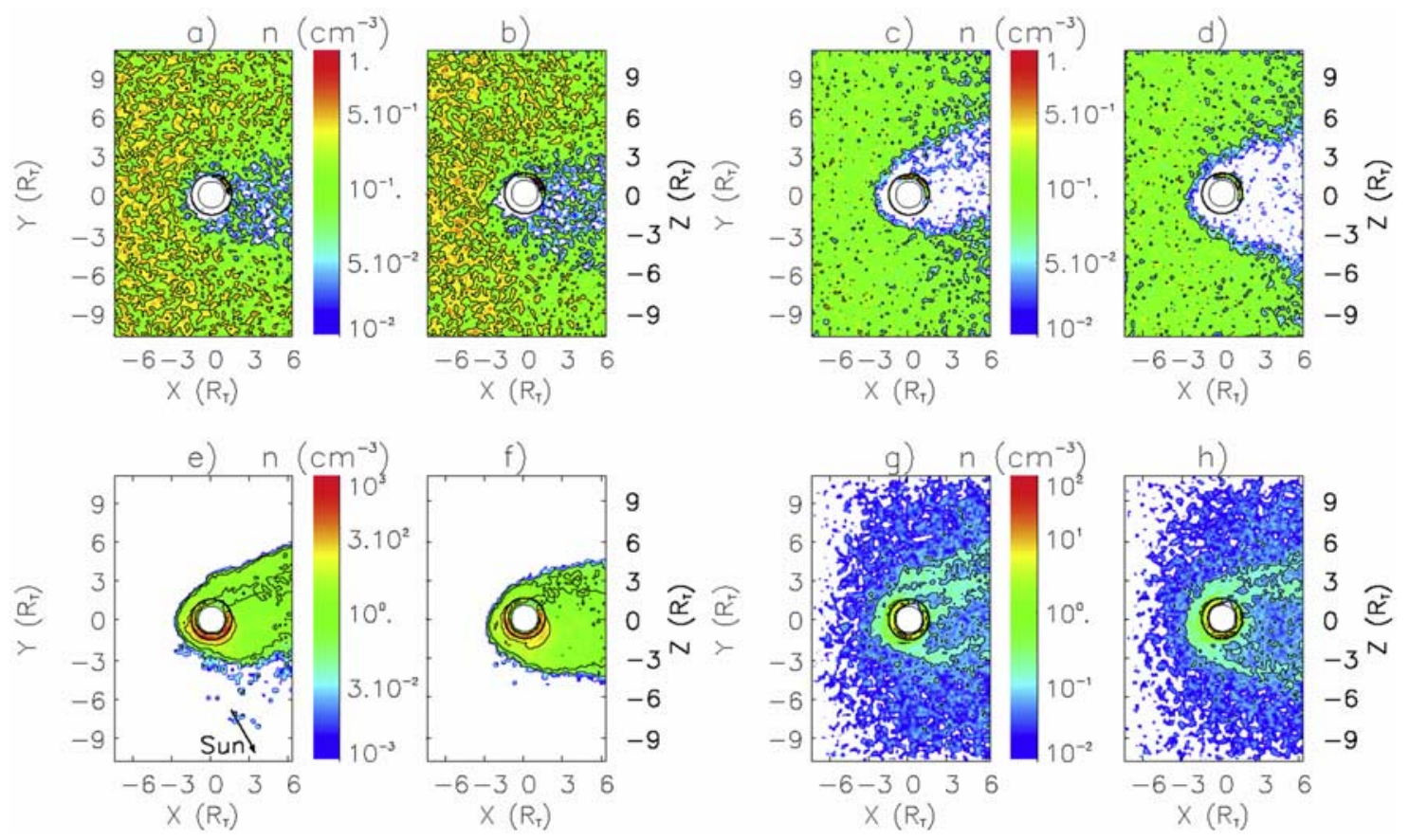

Figure 4. Density maps of (a and $\mathrm{b}$ ) the kronian magnetospheric ions $\mathrm{O}^{+}$ions and ( $\mathrm{c}$ and $\mathrm{d}$ ) low-energy $\mathrm{H}^{+}$, are presented in the XY (Figures 4a and 4c) and XZ (Figures $4 \mathrm{~b}$ and $4 \mathrm{~d}$ ) planes. For these two populations, densities range from $1 . \times 10^{-2}$ ions $\mathrm{cm}^{-3}$ to 1 . ions $\mathrm{cm}^{-3}$. Density maps of the "planetary" species (e and f) $\mathrm{CH}_{4}^{+}$and $(\mathrm{g}$ and $\mathrm{h}) \mathrm{H}_{2}^{+}$are shown in the XY (Figures $4 \mathrm{e}$ and $4 \mathrm{~g}$ ) and $\mathrm{XZ}$ planes (Figures $4 \mathrm{f}$ and $4 \mathrm{~h}$ ). The density scale for $\mathrm{CH}_{4}^{+}$ions vary from $1 . \times 10^{-3}$ ions cm${ }^{-3}$ to $1 . \times 10^{3}$ ions cm $\mathrm{cm}^{-3}$ while the density scale for $\mathrm{H}_{2}^{+}$ions ranges from $1 . \times 10^{-2}$ ions $\mathrm{cm}^{-3}$ to $1 . \times 10^{2}$ ions cm $\mathrm{cm}^{-3}$. Density maps of $\mathrm{N}_{2}^{+}$ions, not shown here, are similar to the density maps of $\mathrm{CH}_{4}^{+}$ions.

factor 100. These two populations behave almost in the same way: they penetrate quite close to the exobase, their densities are depleted by less than a factor 10 in a downstream region aligned with the upstream flow. The depletion of heavy magnetospheric ions in the plasma wake of Titan was first recognized from Voyager observations, although these ions were first thought to be $\mathrm{N}^{+}$ions instead of $\mathrm{O}^{+}$as recently discovered by experiment Cassini/CAPS [Sittler et $a l ., 2005]$. This different behavior is readily explained by the larger Larmor radii of the the $\mathrm{O}^{+}$and energetic protons leading to a deeper penetration in the exosphere of the satellite because they are less affected by the draping the magnetic field which occurs at scales smaller than their gyroradii (the gyroradii for $\mathrm{O}^{+}$, energetic $\mathrm{H}^{+}$and thermal $\mathrm{H}^{+}$ are respectively $5100 \mathrm{~km}, 4100 \mathrm{~km}$, and $343 \mathrm{~km}$ for the set of parameters given in section 2.4). High densities of all magnetospheric ionic species that can be seen below the exobase in Figures $4 \mathrm{a}$ to $4 \mathrm{~d}$ are remnants of the initial stages of the simulation corresponding to the nonphysical transient necessary to build the stationary plasma environment around Titan from a necessarily coarse initial condition. Particles taken to rest by the atmospheric braking are not eliminated from the simulation but they no longer contribute to its time evolution, the unique possible effect of their accumulation below the exobase is to decrease the electric field and thus the variations of the magnetic field in the atmosphere, they indeed play a stabilizing role in this buffer region which cannot be described by an hybrid simulation model.

\subsubsection{Exospheric Plasma}

[30] Density maps of $\mathrm{CH}_{4}^{+}$and $\mathrm{H}_{2}^{+}$in $\mathrm{XY}$ and $\mathrm{XZ}$ planes are presented on Figures $4 \mathrm{e}$ to $4 \mathrm{~h}$. The density maps of $\mathrm{N}_{2}^{+}$ and $\mathrm{CH}_{4}^{+}$being quite similar only the methane ion density maps are shown and discussed. The heavy species $\mathrm{N}_{2}^{+}$and $\mathrm{CH}_{4}^{+}$of the planetary plasma fill the region delimited by the magnetic pileup boundary and colocated with the cavity carved in the flow of low-energy kronian protons.

[31] The magnetic pileup boundary at Titan (defined in the simulation by the maxima of the magnetic field) seems to coincide with a composition boundary separating the heavy species of the planetary plasma from the low-energy component of the kronian plasma. However, the coarse simulation grid may introduce an inaccuracy concerning the colocation of such boundaries. Improved simulations, combined with the Cassini observations, might confirmed or denied this conclusion. The coincidence of the magnetic pileup and composition boundaries has also been observed in the Martian environment [Dubinin et al., 1993] and reproduced by a simulation model [Modolo et al., 2005]. Nevertheless, the situation is less clear-cut in Titan's environment since oxygen ions and energetic protons of the magnetospheric plasma can penetrate the wake of the heavy planetary plasma owing to their very large Larmor radii. The density map of $\mathrm{CH}_{4}^{+}$in the $\mathrm{XY}$ plane displayed in Figure 4e illustrates the shift of the planetary plasma wake toward Saturn, that is, the direction opposite to the corotational electric field. Figure $4 \mathrm{f}$ reveals the asymmetry of the planetary plasma wake with respect to the equatorial plane 


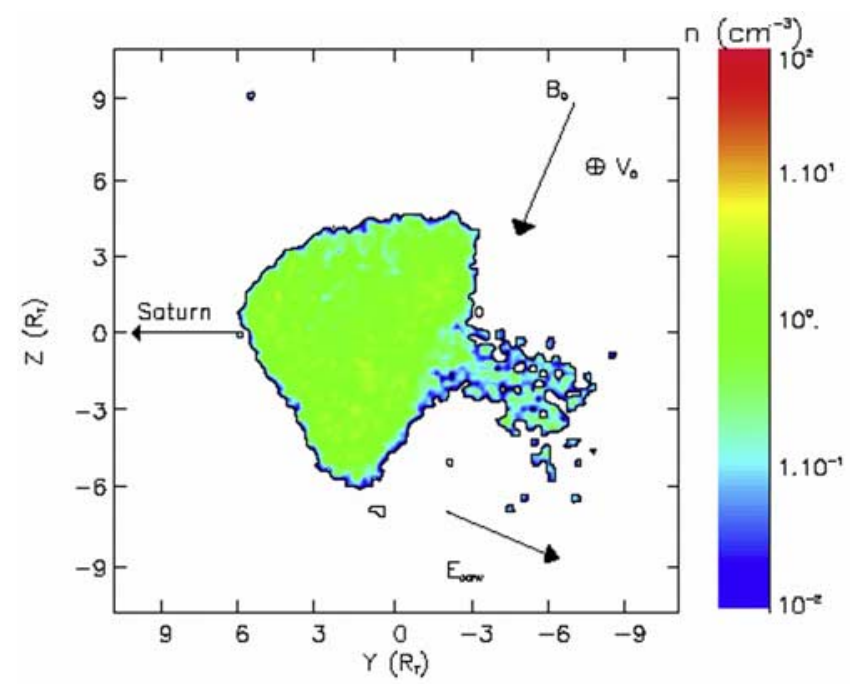

Figure 5. Density map of the $\mathrm{CH}^{+}$in the $\mathrm{YZ}$ plane and located at 6 Titan radii in the wake. The direction of Saturn point toward the $+Y$ direction. The projection of the upstream magnetic field is indicated as well as the direction of the incoming plasma flow.

due to the position of Sun below that plane; the declination of Sun relative to Titan's orbital plane is $\alpha_{\text {sol }}=23.38^{\circ}$. The density reaches a maximum equal to a few hundred of ions per $\mathrm{cm}^{-3}$ below the exobase and on the illuminated side of Titan around the subsolar point (the direction to Sun is indicated by an arrow on Figure 4e). One strength of semikinetic models, compared to fluid models, is to describe the dynamic of each ions species. Such models make possible to monitor the particles behavior and are able to identify the ionization source. The contribution of the different population origin to the global plasma environment is thus feasible (not shown). Photoionization thus appears as the main source of the heavy components of the planetary plasma. A secondary contribution exists on the ramside facing the corotation flow due to electronic impacts. Electronic impact ionization contributes mainly to the creation of the planetary ions detected on the ramside. On the other hand, charge exchange process is a minority ion production for heavy planetary ions. Density of $\mathrm{CH}_{4}^{+}$ and $\mathrm{N}_{2}^{+}$ions produced by charge exchange contributes to less than few ions per $\mathrm{cm}^{-3}$. Meanwhile, these results can be affected by the used of more accurate charge exchange cross sections, and their dependence upon the energy, it is not unrealistic to neglect the charge exchange process for heavier ions $\left(\mathrm{CH}_{4}^{+}\right.$and $\left.\mathrm{N}_{2}^{+}\right)$as a first approximation. This statement can not be applied for light planetary ions $\left(\mathrm{H}_{2}^{+}\right)$. Owing to the low gravity of Titan, the extension of the molecular hydrogen corona is substantial and its coupling with the magnetospheric plasma is important far from Titan $\left(>2 \mathrm{R}_{T}\right)$. Charge exchange process is thus the main ion production of light ions, following by photoionization, and electronic impact ionization.

[32] The wake of the lighter $\mathrm{H}_{2}^{+}$ions is also shifted toward Saturn as evidenced by Figure $4 \mathrm{~g}$, but Figure $4 \mathrm{~g}$ shows no evidence of a north-south asymmetry which could have been expected from photoionization. One noteworthy feature is the presence of the $\mathrm{H}_{2}^{+}$ions far from Titan (up to the edges of the simulated volume at 10 Titan radii) due to the extended corona of molecular hydrogen. $\mathrm{H}_{2}^{+}$ions are the dominant pickup ions population at high altitudes in agreement with Voyager 1 observations [Sittler et al., 2005].

[33] The electron density, equal to the sum of the various ionic densities in an hybrid model, reaches a maximum value of the order of 1000 electrons $\mathrm{cm}^{-3}$, while the Radio and Plasma Wave Science-Langmuir Probe (RPWS-LP) measured a maximum density equal to $3800 \mathrm{~cm}^{-3}$ and $3200 \mathrm{~cm}^{-3}$ for Ta and Tb flybys, respectively, near $1250 \mathrm{~km}$ of altitude [Wahlund et al., 2005]. This important discrepancy can be explained by the absence of a set of chemical equations and a detailed atmosphere in the simulation model, essential to describe the ionosphere of the body. Moreover, the spatial resolution $(500 \mathrm{~km})$, is not sufficient to characterize accurately this region. The detailed ionospheric simulation model developed by Cravens et al. [2005] present a very good agreement with the Cassini's observations. Below the exobase level, the gyroradii of the pickup ions can be much lower than $1000 \mathrm{~km}$ and the flow is more fluid-like, suggesting that a fluid model provides a better description due to a more accurate spatial grid.

[34] The plasma wake of Titan is then mainly composed of heavy pickup ions $\left(\mathrm{CH}_{4}^{+}\right.$and $\left.\mathrm{N}_{2}^{+}\right)$, consistent with the observations of the CAPS experiment [Hartle et al., 2006]. Figure 5 present the $\mathrm{CH}_{4}^{+}$ions density in the wake of Titan at 6 Titan radii from the center of the satellite, in the YZ plane. Two area can be easily distinguished: the main plasma wake and a smaller region in the direction of the corotational electric field. The $\mathrm{CH}_{4}^{+}$density of the central part of the wake reach 4-6 ions per $\mathrm{cm}^{3}$ at 6 Titan radii and is then consistent with the Voyager 1 observations given a density in the wake around $40 \mathrm{~cm}^{-3}$ near 2.8 Titan radii [Gurnett et al., 1982]. The density of $\mathrm{N}_{2}^{+}$ions at 6 Titan radii is slightly lower than the $\mathrm{CH}_{4}^{+}$density. One notable feature is the asymmetric shape of the plasma wake and its shift toward Saturn $(\mathrm{Y}>0)$. In the wake of Titan the simulation results emphasize the importance of the gyroradius effects seen in the Voyager 1 analysis and suggest the use of a 


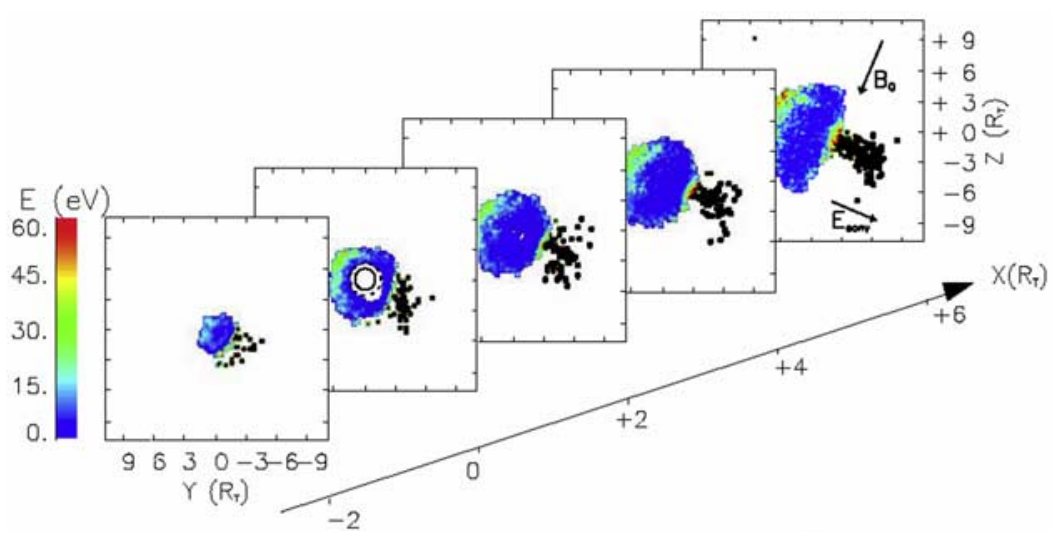

Figure 6. Maps of energy per nucleon for $\mathrm{N}_{2}^{+}$ions in $\mathrm{YZ}$ plane and for different values in the $\mathrm{X}$ directions $(\mathrm{X}=-2,0,+2,+4$ and +6 Titan radii). The color scales vary from 0 to $60 \mathrm{eV}$ per nucleon. The black color indicate ions with an energy greater then $60 \mathrm{eV}$ per nucleon. The incoming plasma flow is aligned with the $\mathrm{X}$ direction. The projection in the $\mathrm{YZ}$ plane of the undisturbed magnetic field, the direction of the corotational electric field (Econv) and the direction of Saturn are displayed.

kinetic approach above $2000 \mathrm{~km}$. The second region, located outside of the main wake of Titan, is strongly extended along the corotational electric field direction. This region contains heavy planetary ions, picked up by the ambient flow, with a significant energy (cf section 3.3). The density of this pickup population is lower by a factor 10 than the ion density in the central part of the wake.

\subsection{Plasma Acceleration}

[35] Hybrid models allow to study the acceleration of the pickup ions, since a kinetic approach is used to describe the ions. Figure 6 shows maps of energy per nucleon for $\mathrm{N}_{2}^{+}$ ions in different plane parallel to the terminator plane (YZ plane). This pseudo 3-D view of the energy allow to characterize the plasma acceleration of the pickup ions in the wake of Titan. Maps of energy for $\mathrm{CH}_{4}^{+}$ions (not seen) present similar features than $\mathrm{N}_{2}^{+}$ions energy maps.

[36] The two region pointed out on the $\mathrm{CH}_{4}^{+}$ions density map (Figure 5) are clearly seen on Figures 6 and 7. In Figure 7, pick-up ions located outside of the plasma wake, are concentrated in the anti-Saturn face, strongly colimated in regards of the corotating electric field.

[37] The maximum energy reach by these pickup ions, in the wake, can reach $400 \mathrm{eV}$ per nucleon and should provide significant signature on the CAPS instrument. Up to now, these high-energy ions (greater than $10 \mathrm{keV}$ ) have not be observed, perhaps due to the trajectory of the Cassini spacecraft which did not cross the pickup ions "beam," or the field of view of the experiment was not in the best position to observe them. In the central part of the wake (Figure 6), the ions are cold (less than $5 \mathrm{eV}$ per nucleon). The acceleration by the corotating plasma is clearly seen, the energy increase progressively from the central part of the wake to the edge of the plasma wake in the direction of the corotational electric field. The region where the corotational electric field point out is a favor region for the acceleration and escape of ions created around the exobase level. When the exospheric ions are born in the corotational electric field side, they are accelerated upward out of the atmopshere and carried away by the kronian flow.

\subsection{Simulation Results Along the Trajectory Orbit}

[38] Figure 8 presents the total density, the speed of the plasma, and the magnetic field components along the trajectory of the spacecraft for plus and minus $30 \mathrm{~min}$ around the closest approach.

[39] The total density (Figure 8a) can be compare with the electron number density estimated by the RPWS-LP measurements [Wahlund et al., 2005]. Meanwhile, this simulation model is very poorly implemented to describe the ionospheric plasma, a satisfactory agreement on the global shape and density values are noticed with the observations. The maximum of the simulated density occurred at 1529 UT and reach the value of $985 \mathrm{~cm}^{-3}$ while observations suggest a maximum of density of $3800 \mathrm{~cm}^{-3}$ at $1528 \mathrm{UT}$. As has been mentioned previously, this model is not adapted to discuss about the Titan's ionosphere since the ionospheric

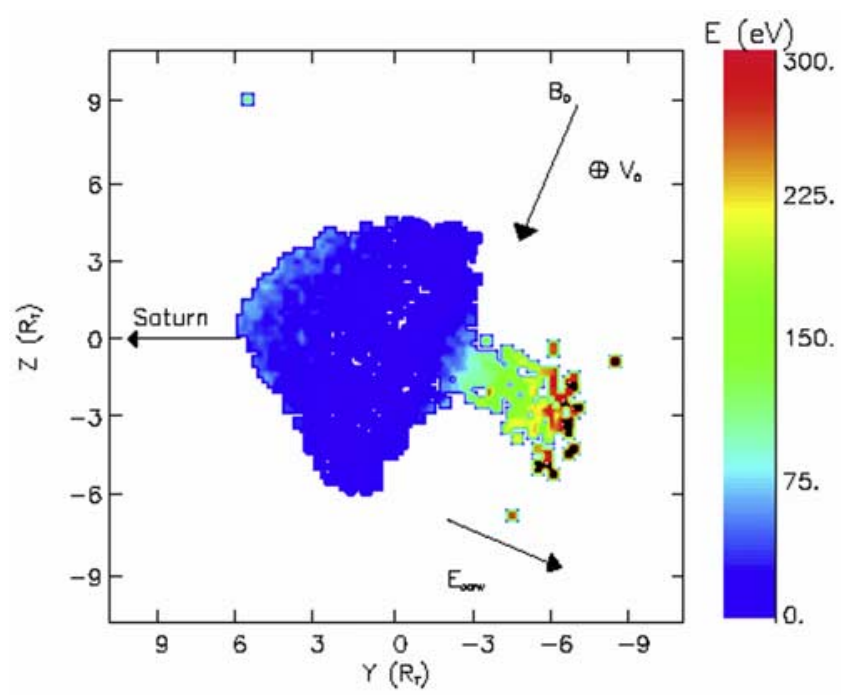

Figure 7. Map of energy per nucleon for $\mathrm{N}_{2}^{+}$ions in the $\mathrm{YZ}$ plane at $\mathrm{X}=+6$ Titan radii. The color scales vary from 0 to $300 \mathrm{eV}$ per nucleon. 


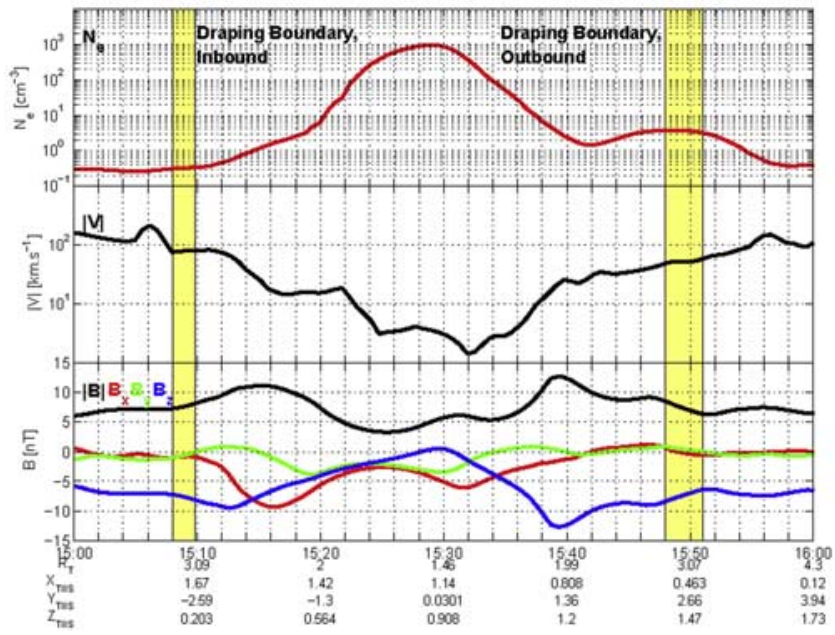

Figure 8. Simulated plasma parameters along the Ta Cassini orbit. (top) The total density, (middle) the plasma speed, and (bottom) the magnetic field components are plotted versus the spacecraft event times. The magnetic field components are presented in the DRAP coordinate system [Neubauer et al., 2006], while the position of the spacecraft is indicating in the TIIS coordinate system [Backes et al., 2005].

chemistry is not included and the spatial resolution is relatively coarse.

[40] Figure $8 \mathrm{~b}$ shows the simulated speed of the plasma and can be compared with the observations made by the Cassini Plasma Spectrometer (CAPS) [Szego et al., 2005]. The deceleration of the plasma flow has been observed from 1508 UT to 1522 UT. This plasma flow deceleration is due to the loading of the magnetospheric flow with heavy planetary ions. The simulation is in excellent agreement with the Cassini observations and predicts a drop of the flow from $100-140 \mathrm{~km} / \mathrm{s}$ to $1-6 \mathrm{~km} / \mathrm{s}$ as has been observed by the Langmuir Probe [Wahlund et al., 2005].

[41] Figure 8c illustrates the magnetic field components along the spacecraft trajectory and can be compared to the magnetic data [Neubauer et al., 2006]. Magnetic field values are presented in the new draping coordinate system DRAP defined by Neubauer et al. [2006]. In this coordinate system $\mathrm{X}_{D R A P}$ is along the flow direction, $\mathrm{Z}_{D R A P}$ is antiparallel to the magnetic field vector $\overrightarrow{B_{0}}$ in the incident flow, and $\mathrm{Y}_{D R A P}$ completes the right handed Cartesian system. This draping coordinate system is chosen to ensure that outside the draping region proper the component $\mathrm{Bx}$, By are essentially zero. According to the nomenclature defined by Neubauer et al. [2006], a fast transition between "weak draping" and "strong draping" is noticed in the simulation at $\sim 1510$ UT, while magnetic field observations show a transition at 1509 UT. The onset and termination have been referred as the draping boundary layers. After the onset, a continuous increase of $\mathrm{Bz}$ is noted in the simulation and the observations. The main point of discrepancies between the model and the observations is the absence in the simulation result of a strong positive $\mathrm{Bx}$ component over the time interval [1535-1539] UT. The coarse simulation grid $(500 \mathrm{~km})$ might be the cause of this issue, since the thickness of the observed structure is $\sim 1200 \mathrm{~km}$ which corresponds to two simulation grid points. The outbound draping boundary in the simulation is noticed at 1548 1551 UT, while observations suggest an outbound boundary at 1541 UT. This difference may be explained by a change in the upstream magnetic field orientation. Meanwhile, the simulation assume a stationary incident flow during the flyby, the magnetic field observations indicate a change between inbound $\left(B_{0}^{i n}=[0.72,2.38,-5.60] \mathrm{nT}\right)$ and outbound $\left(\overrightarrow{B_{0}^{\text {out }}}=[1.99,3.53,-3.93] \mathrm{nT}\right)$ [Backes et al., 2005].

[42] Considering the limited spatial resolution of the present simulation and the possible effect due to the incident plasma flow variations, the simulated plasma environment is in reasonable agreement with the in situ measurements made by spacecraft Cassini.

\subsection{Ion Escaping Flux}

[43] One of the major loss of the neutral exosphere of Titan can be described in terms of plasma escape, where neutral atoms or molecules could be ionized and dragged away. Up to now, no significant data sets are available to give an accurate estimation. Sillanpää et al. [2006] suggest a more or less constant escaping flux for different orbital phase but the electron impact ionization is not included in their model and may affect these estimations. Furthermore the in situ estimation of ion escaping flux is given by a one observation point and it is calculated assuming the geometry of the plasma wake. Global models provide a 3-D view of the plasma environment and take into account the different assymetry of the wake, improving the escaping ion flux estimation.

[44] An estimation of the $\mathrm{N}_{2}^{+}, \mathrm{CH}_{4}^{+}$and $\mathrm{H}_{2}^{+}$ions escaping flux have been derived and presented in Table 4. The estimated loss rates varied by less than $10 \%$ over the time $\mathrm{t} \simeq 1500 \mathrm{~s}$ and time $\mathrm{t}=2600 \mathrm{~s}$. Table 4 presents the average escaping flux over this time period. Since the present simulation model does not present any loss term which can balance the ion production, the escaping flux estimated may be interpreted as a theoretical maximum total ion loss rates.

[45] The ions which leave the simulation box contribute to our estimation of the escaping ions. The major ionization process which contribute to heavy pickup ion escape is the 
Table 4. Estimation of Escaping Flux in Hybrid Simulation for Minimum Solar Condition ${ }^{\mathrm{a}}$

\begin{tabular}{lcccc}
\hline & \multicolumn{4}{c}{ Escaping Flux $\left(\times 10^{25}\right.$ ions s $\left.^{-1}\right)$} \\
\cline { 2 - 5 } & $\mathrm{N}_{2}^{+}$ & $\mathrm{CH}_{4}^{+}$ & $\mathrm{H}_{2}^{+}$ & All Pickup Ions \\
\hline Photoionization & $0.9 \pm 0.1$ & $1.5 \pm 0.1$ & $0.3 \pm 0.0$ & $2.7 \pm 0.2$ \\
Electron impact ion. & $0.4 \pm 0.1$ & $0.7 \pm 0.2$ & $0.1 \pm 0.0$ & $1.2 \pm 0.2$ \\
Charge exchange & $0.0 \pm 0.0$ & $0.1 \pm 0.0$ & $2.1 \pm 0.1$ & $2.2 \pm 0.1$ \\
Total & $1.3 \pm 0.2$ & $2.3 \pm 0.3$ & $2.5 \pm 0.1$ & $6.2 \pm 0.3$ \\
\hline
\end{tabular}

${ }^{\text {a }}$ The escaping flux is detailed following the ionization origin of the exospheric ion.

photoionization, following by the electronic impact ionization and in a minority proportion the charge exchange process, as illustrate Table 4. Owing to the large extension of the molecular hydrogen corona, the charge exchange process between magnetospheric ions and neutrals $\left(\mathrm{H}_{2}\right)$ is the most efficient ionization source of light ions. $\mathrm{H}_{2}^{+}$ions ionized by this process contribute predominantly to the loss rate. As it have been mentioned in section 2.3, charge exchange cross sections used in the simulation model are assumed to be constant and do not vary with the energy. A more accurate estimate of the cross section and their dependence with the energy may affect the estimation of the escaping ions flux with charge exchange process as ionization origin. The total loss rate is in quite good agreement with the estimation deduced from the RPWSLangmuir probe estimation. For the ta flyby, the Langmuir probe measured a total escape flux of $10^{25}$ ions/s, assuming a cylindrical geometry [Wahlund et al., 2005]. This escaping flux is roughly 10 times higher than the loss rate deduced from the Voyager 1 observations [Gurnett et al., 1982] but for a different orbital phase and different solar activity conditions.

[46] The estimation of the heavy ions escaping flux is also in quite good agreement with the estimation given by Sillanpää et al. [2006] from a different hybrid simulation model and suggesting a net ion outflow of the order of $0.45 \times 10^{25} \mathrm{~N}_{2}^{+}$ions per second and $1.12 \times 10^{25} \mathrm{CH}_{4}^{+}$ions per second. Ma et al. [2006] predicts a total escape fluxes less important by a factor 10 and is in better agreement with the Ledvina et al. [2005] estimation. The escaping ion flux can be strongly affected by the orbital phase as well as the usptream parameters of the incoming flow. More Cassini flybys, with global simulations in complements, are necessary to specify if the escaping flux is roughly constant or strongly influence by different parameters.

\section{Conclusions}

[47] A three-dimensional multispecies hybrid model has been designed to investigate the plasma environment of Titan and to estimate the escape of exospheric ions. The model output has been compared to some of the Cassini observations made 26 October 2004 during flyby $T_{A}$ of Titan. The model includes a self-consistent computation of the planetary plasma source thereby allowing an independent estimation of the escape fluxes of three major ionic species. For the first time the full dynamics of six ions species $\left(\mathrm{O}^{+}\right.$, thermal and energetic $\mathrm{H}^{+}, \mathrm{N}_{2}^{+}, \mathrm{CH}_{4}^{+}$, and $\left.\mathrm{H}_{2}^{+}\right)$is included in a global hybrid model.
[48] The distribution of the plasma and the draping of the magnetic field around Titan show a strong asymmetry with respect to the direction of the corotational electric field. We found that the plasma wake of Titan is shifted toward Saturn (i.e., in the direction opposite the corotational electric field) in agreement with Voyager 1 and Cassini observations. In agreement with theoretical predictions, for an incoming submagnetosonic plasma, the simulation does not show any bow shock upstream of Titan but a pileup of the magnetic field lines is clearly visible on the ramside. Other flybys of Titan by Cassini, for various orbital phases of the satellite and different spacecraft trajectories will be investigated and compared to the model output in future investigations with this model.

[49] The presented results emphasize the complex structure of the plasma wake of Titan, dominated by finite gyroradii effects, and point out the necessity of a multispecies approach. This simulation of the plasma interaction at Titan leads to a computed total loss rate of planetary ions equal to $\left(\sim 6 \times 10^{25}\right.$ ions $\left./ s\right)$, a value slightly above the one derived from the observations made by the Langmuir Probe by assuming a cylindral symmetry of the plasma wake.

[50] Acknowledgments. R.M. and G.M.C. wish to acknowledge Michel Blanc for an enlightening discussion about the role of the energetic plasma. They are also strongly indebted to the International Space Science Institute (ISSI) in Bern for the support given to the International Team "Titan, a planetary scale laboratory: Preparing for the Cassini/Huygens exploration" during years 2004-2005 and to the colleagues who participated to the four fruitful meetings organized at ISSI during this period. They are also indebted to the Centre National d'Etude Spatiale (CNES) for their support.

[51] Amitava Bhattacharjee thanks the reviewers for their assistance in evaluating this paper.

\section{References}

Amsif, A., J. Dandouras, and E. C. Roelof (1997), Modeling the production and the imaging of energetic neutral atoms from Titan's exosphere, J. Geophys. Res., 102, 22,169-22,182.

Backes, H., et al. (2005), Titan's magnetic field signature during the first Cassini encounter, Science, 308, 992-995, doi:10.1126/science.1109763.

Berger, M., J. Coursey, M. Zucker, and J. Chang (2005), Stopping-power and range tables for electrons, protons, and helium ions, Natl. Inst. of Stand. and Technol., Gaithersburg, Md. (Available at http://physics.nist. gov/PhysRefData/Star/Text/contents.html)

Birdsall, C. K., and A. B. Langdon (1985), Plasma Physics Via Computer Simulation, McGraw-Hill, New York.

Blanc, M., et al. (2002), Magnetospheric and plasma science with CassiniHuygens, Space Sci. Rev., 104, 253-346.

Brecht, S. H., J. G. Luhmann, and D. J. Larson (2000), Simulation of the Saturnian magnetospheric interaction with Titan, J. Geophys. Res., 105, 13,119-13,130.

Chamberlain, J. W. (1963), Planetary coronae and atmospheric evaporation, Planet. Space Sci., 11, 901-960.

Chanteur, G. M., and R. Modolo (2005), The plasma environment of Titan in the solar wind and in the kronian magnetosphere from global threedimensional hybrid simulations, paper presented at IAGA 2005, Int. Assoc. of Geomagn. and Aeron., Toulouse, France. (Abstract available at http://www.cosis.net/abstracts/IAGA2005/01267/IAGA2005-A01267-1.pdf)

Cravens, T. E., J. U. Kozyra, A. F. Nagy, T. I. Gombosi, and M. Kurtz (1987), Electron impact ionization in the vicinity of comets, J. Geophys. Res., 92, 7341-7353.

Cravens, T. E., C. J. Lindgren, and S. A. Ledvina (1998), A two-dimensional multifluid MHD model of Titans plasma environment, Planet Space Sci., 46, 1193-1205.

Cravens, T. E., et al. (2005), Titan's ionosphere: Model comparisons with Cassini Ta data, Geophys. Res. Lett., 32, L12108, doi:10.1029/ 2005 GL023249.

Dubinin, E., R. Lundin, H. Koskinen, and O. Norberg (1993), Cold ions at the Martian bow shock-PHOBOS observations, J. Geophys. Res., 98, $5617-5623$ 
Eliot, M. (1977), Sections efficaces d'échanges de charge $\sigma_{10}$ et d'ionisation $\sigma_{01}$ d'ions et d'atomes d'hydrogène dans la gamme d'énergie de 1 à $20 \mathrm{keV}$ sur les gaz $\mathrm{N}_{2}, \mathrm{CH}_{4}, \mathrm{C}_{2} \mathrm{H}_{6}, \mathrm{C}_{3} \mathrm{H}_{8}$ et $\mathrm{C}_{4} \mathrm{H}_{10}, J$. Phys., 38, $21-$ 28.

Galand, M., J. Lilensten, D. Toublanc, and S. Maurice (1999), The ionosphere of Titan: Ideal diurnal and nocturnal cases, Icarus, 140, 92-105, doi:10.1006/icar.1999.6113.

Garnier, P., et al. (2007), The exosphere of Titan and its interaction with the kronian magnetosphere: MIMI observations and modeling, Planet. Space Sci., 55, 165-173, doi:10.1016/j.pss.2006.07.006.

Gurnett, D. A., F. L. Scarf, and W. S. Kurth (1982), The structure of Titan's wake from plasma wave observations, J. Geophys. Res., 87, 1395-1403.

Hartle, R. E., E. C. Sittler, K. W. Ogilvie, J. D. Scudder, A. J. Lazarus, and S. K. Atreya (1982), Titan's ion exosphere observed from Voyager 1, J. Geophys. Res., 87, 1383-1394.

Hartle, R. E., et al. (2006), Preliminary interpretation of Titan plasma interaction as observed by the Cassini Plasma Spectrometer: Comparisons with Voyager 1, Geophys. Res. Lett., 33, L08201, doi:10.1029/ 2005GL024817.

Hoffman, J. M., G. H. Miller, and G. J. Lockwood (1982), Charge transfer of ground-state $\mathrm{C}^{+}, \mathrm{N}^{+}$, and $\mathrm{O}^{+}$in $\mathrm{N}_{2}$ and $\mathrm{H}_{2}$, Phys. Rev. A, 25, 19301936, doi:10.1103/PhysRevA.25.1930.

Kabin, K., P. L. Israelevich, A. I. Ershkovich, F. M. Neubauer, T. I. Gombosi, D. L. De Zeeuw, and K. G. Powell (2000), Titan's magnetic wake: Atmospheric or magnetospheric interaction, J. Geophys. Res., 105, $10,761-10,770$.

Kallio, E., I. Sillanpää, and P. Janhunen (2004), Titan in subsonic and supersonic flow, Geophys. Res. Lett., 31, L15703, doi:10.1029/ 2004GL020344

Keller, C. N., T. E. Cravens, and L. Gan (1994), One-dimensional multispecies magnetohydrodynamic models of the ramside ionosphere of Titan, J. Geophys. Res., 99, 6511-6525.

Keller, C. N., V. G. Anicich, and T. E. Cravens (1998), Model of Titans ionosphere with detailed hydrocarbon ion chemistry, Planet. Space Sci., 46, $1157-1174$

Kim, Y. K., et al. (2004), Electron-impact ionization cross section database (version 3.0), Natl. Inst. of Stand. and Technol., Gaithersburg, Md.

Krimigis, S. M., J. F. Carbary, E. P. Keath, T. P. Armstrong, L. J. Lanzerotti, and G. Gloeckler (1983), General characteristics of hot plasma and energetic particles in the Saturnian magnetosphere: Results from the Voyager spacecraft, J. Geophys. Res., 88, 8871-8892.

Krimigis, S. M., et al. (2004), Magnetosphere Imaging Instrument (MIMI) on the Cassini Mission to Saturn/Titan, Space Sci. Rev., 114, 233-329, doi:10.1007/s11214-004-1410-8.

Lebonnois, S., D. Toublanc, F. Hourdin, and P. Rannou (2001), Seasonal variations of Titan's atmospheric composition, Icarus, 152, 384-406, doi:10.1006/icar.2001.6632.

Ledvina, S. A., and T. E. Cravens (1998), A three-dimensional MHD model of plasma flow around Titan, Planet. Space Sci., 46, 1175-1191.

Ledvina, S. A., T. E. Cravens, and K. Kecskeméty (2005), Ion distributions in Saturn's magnetosphere near Titan, J. Geophys. Res., 110, A06211, doi:10.1029/2004JA010771.

Lindsay, B. G., R. L. Merrill, H. C. Straub, K. A. Smith, and R. F. Stebbings (1998), Absolute differential and integral cross sections for charge transfer of $\mathrm{keV} \mathrm{O}{ }^{+}$with $\mathrm{N}_{2}$, Phys. Rev. A, 57, 331-337, doi:10.1103/ PhysRevA.57.331.

Luhmann, J. G. (1996), Titan's ion exosphere wake: A natural ion mass spectrometer?, J. Geophys. Res., 101, 29,387-29,393.

Ma, Y., A. F. Nagy, T. E. Cravens, I. V. Sokolov, K. C. Hansen, J.-E. Wahlund, F. J. Crary, A. J. Coates, and M. K. Dougherty (2006), Comparisons between MHD model calculations and observations of Cassini flybys of Titan, J. Geophys. Res., 111, A05207, doi:10.1029/ 2005JA011481.

Matthews, A. (1994), Current advance method and cyclic leapfrog for 2d multispecies hybrid plasma simulations, J. Comput. Phys., 112, $102-$ 116.

Maurice, S., E. C. Sittler, J. F. Cooper, B. H. Mauk, M. Blanc, and R. S. Selenick (1996), Comprehensive analysis of electron observations at Saturn: Voyager 1 and 2, J. Geophys. Res., 101, 15,211-15,232.

Modolo, R. (2004), Modélisation de l'interaction du vent solaire, ou du plasma kronien, avec les environnements neutres de Mars et de Titan, Ph.D. thesis, CETP-IPSL and Univ. de Versailles Saint-Quentin en Yvelines, Versailles, France.

Modolo, R., and G. M. Chanteur (2005), Modélisation de l'interaction du vent solaire avec l'environnement neutre de Mars, Not. du Pôle de Planétol. 13, Inst. Pierre Simon Laplace, Paris. (Available at http:// www.ipsl.jussieu.fr/documentation/NotesPlaneto/Notes.htm).
Modolo, R., G. M. Chanteur, E. Dubinin, and A. P. Matthews (2005), Influence of the solar EUV flux on the Martian plasma environment, Ann. Geophys., 23, 433-444.

Modolo, R., G. M. Chanteur, E. Dubinin, and A. P. Matthews (2006), Simulated solar wind plasma interaction with the Martian exosphere: Influence of the solar EUV flux on the bow shock and the magnetic pile-up boundary, Ann. Geophys., 24, 3403-3410.

Nagy, A. F., Y. Liu, K. C. Hansen, K. Kabin, T. I. Gombosi, M. R. Combi, D. L. DeZeeuw, K. G. Powell, and A. J. Kliore (2001), The interaction between the magnetosphere of Saturn and Titan's ionosphere, J. Geophys. Res., 106, 6151-6160.

Ness, N. F., M. H. Acuna, and K. W. Behannon (1982), The induced magnetosphere of Titan, J. Geophys. Res., 87, 1369-1381.

Neubauer, F. M. (1992), Titan's magnetospheric interaction, in Symposium on Titan, edited by B. Kaldeich, Eur. Space Agency Spec. Publ, ESA SP$338,267-272$.

Neubauer, F. M., D. A. Gurnett, J. D. Scudder, and R. E. Hartle (1984), Titan's magnetospheric interaction, in Saturn, edited by T. Gehrels and M. Shapley Matthews, pp. 760-787, Univ. of Arizona Press, Tucson.

Neubauer, F. M., et al. (2006), Titan's near magnetotail from magnetic field and electron plasma observations and modeling: Cassini flybys TA, TB, and T3, J. Geophys. Res., 111, A10220, doi:10.1029/2006JA011676.

Phaneuf, R. A., F. W. Meyer, and R. H. McKnight (1978), Single-electron capture by multiply charged ions of carbon, nitrogen, and oxygen in atomic and molecular hydrogen, Phys. Rev. A, 17, 534-545, doi:10.1103/PhysRevA.17.534.

Rees, M. H. (1989), Physics and Chemistry of the Upper Atmosphere, 304 pp., Cambridge Univ. Press, Cambridge, U.K.

Richards, P. G., J. A. Fennelly, and D. G. Torr (1994), EUVAC: A solar EUV flux model for aeronomic calculations, J. Geophys. Res., 99, 89818992.

Schunk, R. W., and A. F. Nagy (2000), Ionospheres, Physics, Plasma Physics and Chemistry, Cambridge Univ. Press, Cambridge, U.K.

Sillanpää, I., E. Kallio, P. Janhunen, W. Schmidt, K. Mursula, J. Vilpolla, and P. Tanskanen (2006), Hybrid simulation study of ion escape at Titan for different orbital positions, Adv. Space Res., 38, 799-805.

Simon, S., A. Bößwetter, U. Motschmann, and K. H. Glassmeier (2006), Plasma environment of Titan: A 3-D hybrid simulation study, Ann. Geophys., 24, 1113-1135.

Sittler, E. C., R. E. Hartle, A. F. Viñas, R. E. Johnson, H. T. Smith, and I. Mueller-Wodarg (2005), Titan interaction with Saturn's magnetosphere: Voyager 1 results revisited, J. Geophys. Res., 110, A09302, doi:10.1029/ 2004JA010759.

Szego, K., et al. (2005), The global plasma environment of Titan as observed by Cassini Plasma Spectrometer during the first two close encounters with Titan, Geophys. Res. Lett., 32, L20S05, doi:10.1029/ 2005GL022646.

Toublanc, D., J. P. Parisot, J. Brillet, D. Gautier, F. Raulin, and C. P. McKay (1995), Photochemical modeling of Titan's atmosphere, Icarus, 113, 2 26, doi:10.1006/icar.1995.1002.

Vervack, R. J., B. R. Sandel, and D. F. Strobel (2004), New perspectives on Titan's upper atmosphere from a reanalysis of the Voyager 1 UVS solar occultations, Icarus, 170, 91-112, doi:10.1016/j.icarus.2004.03.005.

Wahlund, J.-E., et al. (2005), Cassini measurements of cold plasma in the ionosphere of Titan, Science, 308, 986-989, doi:10.1126/ science. 1109807 .

Waite, J. H., et al. (2005), Ion neutral mass spectrometer results from the first flyby of Titan, Science, 308, 982-986, doi:10.1126/ science. 1110652 .

Wolf, D. A., and F. M. Neubauer (1982), Titan's highly variable plasma environment, J. Geophys. Res., 87, 881-885.

Yelle, R. V., N. Borggren, V. de La Haye, W. T. Kasprzak, H. B. Niemann, I. Müller-Wodarg, and J. H. Waite (2006), The vertical structure of Titan's upper atmosphere from Cassini Ion Neutral Mass Spectrometer measurements, Icarus, 182, 567-576, doi:10.1016/j.icarus.2005.10.029.

Yung, Y. L. (1987), An update of nitrile photochemistry on Titan, Icarus, 72, 468-472, doi:10.1016/0019-1035(87)90186-2.

Yung, Y. L., M. Allen, and J. P. Pinto (1984), Photochemistry of the atmosphere of Titan: Comparison between model and observations, Astrophys. J. Suppl., 55, 465-506, doi:10.1086/190963.

G. M. Chanteur, Swedish Institute of Space Physics, Uppsala Division, Box 537, SE-751 21 Uppsala, Sweden.

R. Modolo, Centre d'Etudes des Environnements Terrestre et Plantaires, Institut Pierre Simon Laplace, 10-12 avenue de l'Europe, F-78140 Vélizy, France. (ronan.modolo@cetp.ipsl.fr) 